\title{
Magnetic resonance angiographic assessment after extracranial-intracranial bypass surgery
}

\author{
S S Praharaj, A Coulthard, A Gholkar, P English, A D Mendelow
}

\begin{abstract}
Extracranial-intracranial (EC-IC) bypass operation may be performed to augment the distal cerebral circulation. The bypass patency is usually assessed postoperatively with conventional cerebral angiography. Six patients are reported in whom the bypass patency was assessed using magnetic resonance angiography (MRA): Two had intracavernous carotid aneurysms, three had base of skull tumours encompassing the internal carotid artery, and one had occlusion of the right internal carotid artery with poor collateral reserve as confirmed by an acetazolamide stimulation test. Postoperative conventional cerebral angiography was also obtained in three patients for comparison. The MRA correlated reasonably well with conventional cerebral angiography in showing bypass patency, although conventional cerebral angiography was superior in demonstrating small vessels; MRA has the added advantage of showing the relative flow contribution and flow direction under physiological conditions by using targeted presaturation pulses.
\end{abstract}

Department of Surgery (Neurosurgery), The Medical School,

University of

Newcastle upon Tyne,

Framlington Place,

Newcastle upon Tyne,

UK

S S Praharaj

A D Mendelow

Department of

Radiology, Royal

Victoria Infirmary,

Newcastle upon Tyne,

UK

A Coulthard

A Gholkar

P English

Correspondence to:

Professor A D Mendelow,

Department of

Department of

Neurosurgery, Ward-31,

North Wing, Newcastle

General Hospital, Westgate

RE4 6BE, UK.

Received 11 April 1995

and in revised form

11 October 1995

Accepted 8 November 1995 the patency of the EC-IC anastomosis postoperatively with conventional angiography is not without risks. The procedure is invasive, requires admission to hospital, and has a well recognised morbidity which is increased if selective catheterisation is required. ${ }^{4}$ Magnetic resonance angiography (MRA) is a noninvasive outpatient procedure for visualising the cerebral vessels. It also provides the unique advantage of assessing the direction of blood flow and the relative flow contribution by the extracranial supply under physiological circumstances (as opposed to the anatomical patency by forcible dye injection as seen in conventional angiography) by manipulating the scanning sequences.

In this study we have evaluated the role of MRA in assessing the patency and flow dynamics of EC-IC anastomosis postoperatively.

\section{Materials and methods}

Patients attended for outpatient MR angiographic assessment (Magnetom Impact 1.0 T, Siemens). A 3D time of flight sequence was used with magnetisation transfer contrast (MTC) and tilt optimised non-saturating excitation (TONE).

Two consecutive MRA sequences were acquired. Firstly, a 64 partition three dimensional imaging volume was placed at the circle of Willis, care being taken to encompass the site of anastomosis. This sequence was acquired axially with a transaxial presaturation band placed above the imaging volume across the sagittal sinus to remove flow signal from the venous blood. For the second study an additional sagittally oriented presaturation band was placed over the circle of Willis and basilar arteries, to remove all signal contribution from arterial blood flowing in the conventional direction (medially to laterally). A maximum intensity projection algorithm was then used to reconstruct angiographic images from the two data sets. The first sequence produced an arterial study: the second sequence produced a study derived from blood flowing laterally to medially - that is, via the EC-IC anastomosis.

On one patient (No 6), the technique was modified to include a sequence which allowed subtraction of background signal, improving small vessel visualisation. 


\section{CASE HISTORIES}

Patient 1

This thirty three year old patient bled from a giant cavernous segment aneurysm of the left internal carotid artery. Angiography showed congenital narrowing of the right internal carotid artery in addition to the giant left internal carotid artery cavernous segment aneurysm. She underwent right superficial temporal to middle cerebral artery (STAMCA) anastomosis and ligation of the left internal carotid artery. Postoperative conventional angiography showed a patent right STA-MCA bypass. Assessment with MRA one year after operation showed a patent anastomosis with flow within the branches of the distal middle cerebral artery. These findings were confirmed on conventional angiography.

\section{Patient 2}

This forty one year old man had recurrent right hemispheric transient ischaemic attacks. Conventional angiography showed complete occlusion of the right internal carotid artery. Cerebral blood flow studies after acetazolamide stimulation confirmed poor collateral reserve to the right hemisphere. A right superficial temporal-middle cerebral artery bypass was performed after which he made an uneventful recovery. Assessment with MRA three years after operation showed filling of the anterior, middle, and posterior cerebral arteries on the right. Saturation of the internal carotid artery and basilar artery contribution showed flow within the EC-IC anastomosis and distal right middle cerebral artery. Conventional angiography confirmed a widely patent EC-IC bypass with good filling of the right middle cerebral artery.

\section{Patient 3}

A forty seven year old man had recurrent left middle cerebral artery territory transient ischaemic attacks, and a complete occlusion of the left internal carotid artery. He also had a tentorial meningioma in close association with the occluded internal carotid artery. $\mathrm{He}$ underwent EC-IC anastomosis with total exci-

Figure 1 MRA study showing the occluded right cavernous internal carotid artery aneurysm; the proximal right middle cerebral artery is

demonstrated, and the ECIC anastamosis can be seen laterally.

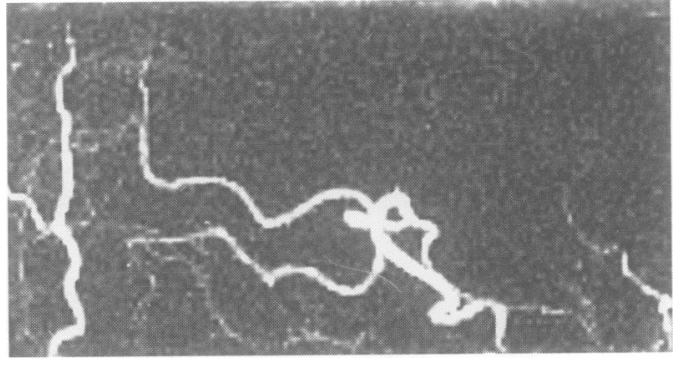

Figure 2 Oblique projection of MRA with a presaturation band showing the signal contribution from the patent EC-IC anastomosis to branches of the distal middle cerebral artery.

sion of the meningioma and made an excellent postoperative recovery.

Postoperative MRA showed a patent EC-IC anastomosis.

\section{Patient 4}

A twenty seven year old man presented with right sided retro-orbital pain, headache, and diplopia. He underwent conventional angiography for a suspected aneurysm but was found to have a right sphenoid wing meningioma. In view of the internal carotid artery feeders and the risk of a hemispheric infarct if the internal carotid artery was to be compromised, he underwent EC-IC bypass with clamping of the right internal carotid artery followed five days later by internal carotid artery ligation. An initial MRA study showed good filling of the anterior, middle, and posterior cerebral arteries bilaterally. Removal of the carotid and basilar contribution showed flow within a patent anastomosis.

\section{Patient 5}

A forty year old woman presenting with diplopia was found on CT to have a meningioma over the cavernous sinus on the right side, with extrinsic compression of the intracavernous internal carotid artery. She underwent EC-IC bypass on the right side followed several days later by removal of the tumour, with preservation of the internal carotid artery. An MRA study clearly demonstrated the anterior, middle, and posterior cerebral arteries bilaterally. After removing the contribution from the carotid and basilar circulation the anastomosis was shown to be patent but only a few small distal middle cerebral artery branches were seen. This was because it had not been necessary to ligate the internal carotid artery as had originally been anticipated.

\section{Patient 6}

This 56 year old woman presented with severe right periorbital pain and diplopia (due to a right sixth nerve paresis) and subsequent CT and angiography confirmed the diagnosis of an aneurysm of the intracavernous carotid artery on the right side. She underwent an EC-IC bypass because a test balloon occlusion produced a transient hemiparesis; MRA was obtained 49 days after the operation (fig 1). The EC-IC anastomosis was patent, and 
Figure 3 Conventional immediate postoperative selective DSA with similar projection to fig 2 for comparison.

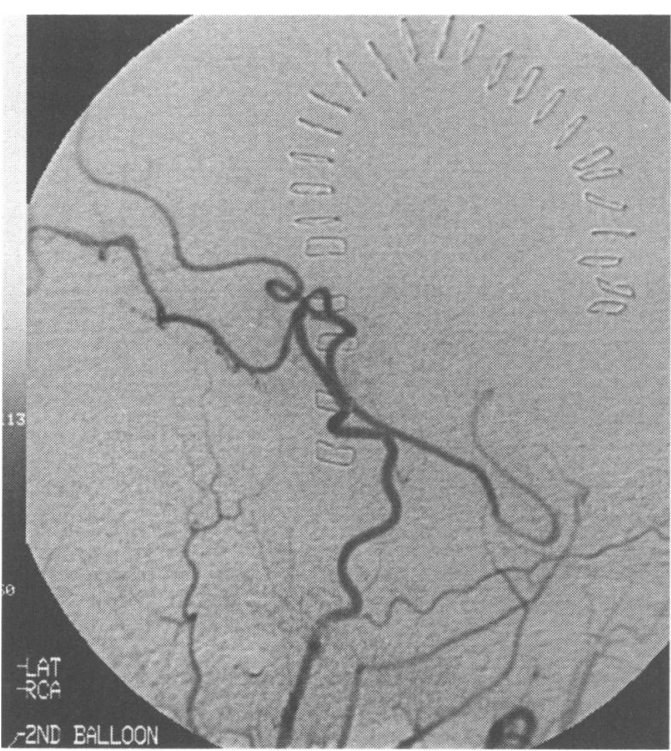

seemed to contribute significantly to the distal middle cerebral artery branch filling (fig 2). The patent anastomosis has been confirmed on conventional angiography (fig 3).

\section{Discussion}

EC-IC bypass has been used for several different ischaemic and potentially ischaemic disorders of the brain. The operation declined in popularity for patients with occlusive stroke after the international cooperative study of extracranial-intracranial arterial anastomosis in 1985 showed that it did not reduce the rate of stroke and stroke related deaths in patients with carotid or middle cerebral artery occlusions or stenosis. ${ }^{1}$ However, patient selection by evaluation of the collateral circulation on angiogram, impaired regional cerebral blood flow, and reserve capacity ${ }^{5}$ was not evaluated in the EC-IC bypass study. Its role under these circumstances therefore remains uncertain. Furthermore, the operation may be of value in providing a collateral supply when intracranial vessels are intentionally occluded in the treatment of giant aneurysms, tumours, or in Moya Moya disease. ${ }^{2}$ Current patency rates for this procedure as established by conventional angiography are over $96 \% .^{12}$ Conventional angiography, however, is an invasive procedure with established morbidity including embolic strokes, vessel dissection, groin haematoma, and infection. ${ }^{4}$ The technique only shows vessels downstream from the catheter tip and projections have to be chosen prospectively. If additional projections are required, additional injections of contrast agent are necessary. After conventional angiography the patient is usually confined to bed for some hours and the procedure is most often performed on inpatients.
Magnetic resonance angiography is a noninvasive outpatient procedure which shows all the vessels within the imaging volume without the need for intravenous contrast agents. A single acquisition can be reformatted in any projection plane retrospectively. Evaluation of EC-IC bypass with MRA has been attempted before $^{6}$ using conventional time of flight sequences: these correlated reasonably well with conventional angiography in terms of patency but did not provide information on the flow direction. Our study applied selective presaturation pulses to the time of flight MRA study to obtain additional information on the flow direction in patients who had undergone EC-IC anastomosis. Our experience suggests that the technique can play an important part in these patients. The three studies described in which the patients also underwent conventional angiography have shown, as in other applications of this technique, that there is a good correlation between conventional angiographic and MRA findings.

In one study (patient 5) the MRA showed rather poor flow in the vessels distal to the anastomosis after saturation of the flow contribution from the internal carotid artery. The reason may be that the internal carotid artery (on the same side) had not been occluded as had originally been planned, and hence the flow contribution from the bypass to the distal middle cerebral artery vessels was proportionately low. Thus interestingly, MRA using the time of flight technique with targeted presaturation band reflects the flow patterns and relative flow contributions in the physiological state as opposed to the anatomical patency shown on forcible dye injection during conventional angiography.

Time of flight MR angiography is now becoming widely available. Our experience suggests that the technique we have described can usefully determine whether EC-IC anastomosis is patent or not. The MRA technique has the advantage of being non-invasive and can be performed on outpatients.

1 The EC-IC Bypass Study Group: Failure of extracranialintracranial arterial bypass to reduce the risk of ischaemic stroke. Results of an international randomised trial. $N$ Engl F Med 1985;313:1191.

2 Amine AR, Moody RA, Meeks W. Bilateral superficial temporal-middle cerebral artery anastomosis for MoyaMoya syndrome. Surg Neurol 1977;8:3-6.

3 Oppel F, Schramm J, Bradac GB, Brock M. Extracranialintracranial bypass surgery: correlation between longterm results and angiographic findings. In: Brihaye J term results and angiographic findings. In: Brihaye $\mathrm{J}$, Vigouroux R, Achslogh , et al, eds. 7th Europ
of neurosurgery. Brussels: EANS, 1983:222.

4 Hankey GJ, Warlow CP, Sellar RJ. Cerebral angiographic risk in mild cerebrovascular disease. Stroke 1990;21: 209-22.

5 Mendelow AD, Patterson J. Recovery of cerebrovascular reserve capacity following extracranial-intracranial bypass surgery in man. In: Callaghan N, Galvin R, eds. Recen research in neurology. London: Pitman, 1984:89-93,

6 Kodama T, Ueda J, Suzuki Y, Yano T, Watanabe K. MRA in the evaluation of EC-IC bypass patency. $f$ Comput Assist Tomogr 1993;17:922-6. 